the ether, whose density is function of the velocity of propagation of light and heat, as also of the wave-lengths.

Multiplying all these masses by the square of the velocity of each particle relatively to the centre of gravity of the solar system, we obtain the factor $\frac{1}{2} \mathrm{M} v^{2}=$ the total kinetic energy of the solar system.

This constant kinetic energy (if the second hypothesis be admitted, in which $\frac{1}{2} m v^{2}$ is constant) is not distributed throughout the solar system in a regular and fixed manner. Sometimes a planet, as Jupiter, is at the extremity of the larger axis of his ellipse, and advances more slowly; sometimes, on the contrary, his velocity is accelerated and passes through a maximum to another position of his orbit.

At the same minute all the planets are revolving round the sun, some with their maximum velocity, others with their minimum velocity, others, again, with intermediate velocities. We may make addition of all these kinetic energies of the whole solar system, and differentiate the total equation with reference to time. The variations thus obtained for each hour will naturally eliminate all the quantities of constant kinetic energy represented by the rotaticn of the stars on their own axes ; they will merely show the increase or the diminution of the whole of the variable kinetic energies of the svstem.

One may easily draw a curve of these variations calculated by the ephemerides of the principal planets. Jupiter will play a preponderating rôle in this calculation.

Considering still the second hypothesis, in which the attraction is merely the result of shocks, it is evident that the attraction manifested by each planet for the bodies which are on its surface will be the echo of the kinetic energy disposable on this planet. This kinetic energy will be variable according to the day and hour of observation.

In fact, the kinetic energy of the solar system being fixed and constant, if the planets, on a certain day, absorb into their own mass a maximum quantity of kinetic energy, the cause of gravity on the earth will be diminished by the whole of the excess which is accumulated in these bodies in motion, and the acceleration $g$ will pass through a minimum. On the other hand, when, a few years later, the whole of the planets give a minimum total of kinetic energy for their masses in motion, the value of $g$, for the same reasons, must pass through a maximum.

It is easily understood that the value of the terrestrial attraction cannot remain con tant if the disposable kinetic energy varies in function of the time and of the respective position of the other planets.

Now, we may calculate the total mass $M$ of the system, the partial masses and their variable velocities; we obtain for these variations considerable values; then if we register carefully the values of $g$ obtained directly. during observations which must continue at the least several years, and if we trace a curve of the values of $g$ so obtained, we should find the following coincidence :-

The curve of variations of the total kinetic energy of the planets must be inverse to the curve of values of $g$ referred to the same time.

The differences between the maxima and the minima of the two curves, taken on the same ordinate, will give the measure of the velocity of propagation of the kinetic energy in the ether of the solar system.

These conclusions are rigorous in the case of the hypothesis,

$$
\frac{1}{2} m v^{2}=\text { constant, }
$$

being in accurdance with nature.

In the case, on the other hand, of attraction being an essential property of matter, and of our having-

$$
\frac{1}{2} m v^{2}+\text { the potential }=\text { constant, }
$$

we should find for $g$ a constant, since $g$ is the sole manifestation of a constant potential, supposing the mass of the earth is constant during the course of the observations of $g$.

It will be necessary, then, to take account of perturbations of the moon for the measurements of $g$, as also of those of the sun, then to verify whether, these corrections having been made, $g$ is constant.

I believe that this experimental method is the only means we possess of diagnosticating with certainty on the essential properties of matter, and of deciding between those two great theories which are both maintained by men of incontestable merit.

As to the measurement of $g$, there are several oferative processes, and it will be indispensable, before commencing observations, to discuss analytically the advantages of each of them, and the modes of inscription of the values obtained.
The optical means of registration, the mechanical actions connected with the motion of pendulums, and the kind of pendulums, will be so many important subjects of discussion, in the case of taking these researches in hand, which I consider as very useful for the definitive settlement of physical theories.

This is a rather long letter, you see, dear Teacher; but I thought to explain to you the object which I pursue, in its general traits, happy indeed if the experiments may be undertaken under your benevolent auspices.

Accept, dear Teacher, I pray you, the expression of my gratitude and entire devotion. Raoul Pictet

\section{A GEOMETRICAL CONSTRUCTION GIVING THE RELATTON BETWEEN THE WASTE AND USEFUL WORK IN A SHUNT DYNAMO}

THE ratio between the portion of electrical energy utilisable in the external circuit of a shunt dynamo and the portion wasted in heating the wire of the armature and field magnet is easily calculated as soon as one knows the resistances of the armature and magnet wires, and the resistance equivalent to the external circuit; and I do not know that there is any great advantage in putting it into a geometrical form. Still there are people who prefer a cunstruction to a formula, and the following construction is easily made, especially with the use of squared paper.

In the figure annexed, let o $\mathrm{A}$ represent the resistance of the armature between the points where the branching occurs; o $B$

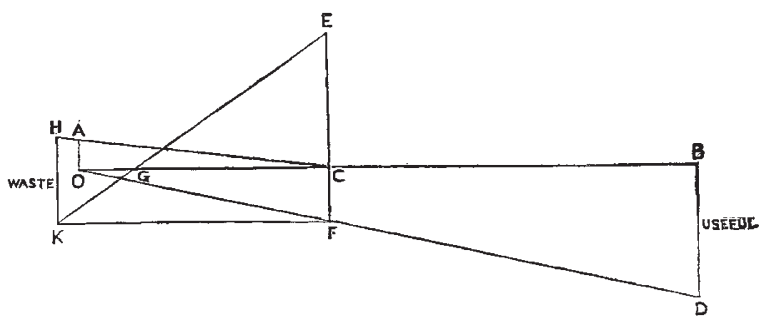

the resistance of the field magnet wire; and $\mathrm{OC}$ the resistance of the external circuit, or its equivalent.

Erect lines to represent the useful work (E.M.F. $\times$ current), on any convenient scale, at $\mathrm{C}$ and at $\mathrm{B}$; viz. $\mathrm{C} E$ and $\mathrm{B} D$.

Join $\mathrm{O} D$, producing $\mathrm{EC}$ to meet it at $\mathrm{F}$.

Lay off C G equal to E F ; draw E G and a horizontal through F. Then from their meeting point $\mathrm{K}$ draw a vertical, meeting $\mathrm{C} A$ in $\mathrm{H}$.

The length $\mathrm{HK}$ so determined represents the waste portion of the total electrical energy, on the same scale as $B D$ or E C represents the useful.

In this figure the effect of the armature resistance in tilting up the line $\mathrm{CA}$ and so increasing the waste is very manifest; the increase of waste by decreasing the resistance $O B$ is somewhat less striking, but quite distinct ; the effect of a change in $\mathrm{OC}$ is, as it should be, not so obvious. It may be noted that the most economical value for $\mathrm{OC}$ is very nearly indeed a geometric mean between $\mathrm{OA}$ and $\mathrm{OB}-\mathrm{OA}$; which is an easy rule to apply in practice.

Liverpool, July I9

OLIVER J. LODge

\section{UNIVERSITY AND EDUCATIONAL INTELLIGENCE}

THE following is the list of candidates successful in the competition for the Whitworth scholarships, 1882, in connection with the Science and Art Department :-Charles Webster, apprentice; John H. Tomlinson, apprentice ; James M. Beaman, fitter; Thomas Turner, engineer; D. Codrington Selman, engineer; Charles B. Outon, draughtsman; George $H$. Banister, draughtsman; Frederick Lane, fitter; William D. Laird, engine fitter; Joseph Parry, engine fitter; Albert F. Ravenshear, apprentice; Charles W. Carter, brass-finisher; Alfred Barrow, fitter ; Henry C. King, fitter ; Malcolm Douglas, apprentice ; Thomas H. Gardner, engineer ; Ernest E. Haine, engineer; George Halliday, engineer; George W. Buckwell, draughtsman ; Louis H. Cochrane, engineer; William Duncanson, engine fitter; Henry Brown, engineer; William T. Hatch, 